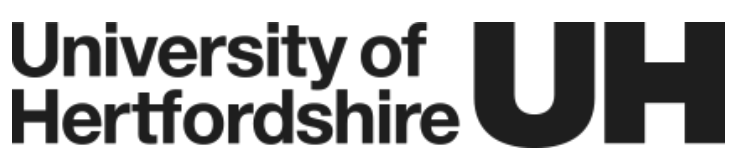

Citation for the published version:

Wright, H., Wellsted, D., Gratton, J., Besser, S. J., \& Midgley, N. (2019). Use of the Strengths \& Difficulties Questionnaire to identify treatment needs in looked after children referred to CAMHS. Developmental Child Welfare. DOI:

$10.1177 / 2516103218817555$

Document Version: Accepted Version

Link to the final published version available at the publisher:

https://doi.org/10.1177/2516103218817555

Copyright (C) 2019 The Authors. Reprinted by permission of SAGE Publications.

\section{General rights}

Copyright $\odot$ and Moral Rights for the publications made accessible on this site are retained by the individual authors and/or other copyright owners.

Please check the manuscript for details of any other licences that may have been applied and it is a condition of accessing publications that users recognise and abide by the legal requirements associated with these rights. You may not engage in further distribution of the material for any profitmaking activities or any commercial gain. You may freely distribute both the url (http://uhra.herts.ac.uk/) and the content of this paper for research or private study, educational, or not-for-profit purposes without prior permission or charge.

\section{Take down policy}

If you believe that this document breaches copyright please contact us providing details, any such items will be temporarily removed from the repository pending investigation.

\section{Enquiries}

Please contact University of Hertfordshire Research \& Scholarly Communications for any enquiries at rsc@herts.ac.uk 


\title{
Use of the Strengths \& Difficulties Questionnaire to identify treatment needs in looked after children referred to CAMHS
}

\author{
Hannah Wright ${ }^{1}$, David Wellsted ${ }^{2}$, Jacqui Gratton ${ }^{1}$, Sarah Jane Besser ${ }^{2}$, and Nick \\ Midgley $^{34}$
}

\begin{abstract}
Background: In England and Wales, the single-informant Strengths \& Difficulties Questionnaire (SDQ) is used to assess and monitor looked after children's (LAC) mental health; and some targeted Child \& Adolescent Mental Health Services (CAMHS) include a minimum SDQ score in their acceptance criteria. However, its ability to identify LAC who need mental health treatment is insufficiently understood.

Methods: 144 LAC referrals to a Targeted CAMHS Team were screened as part of a larger study. To establish how well the SDQ identified children who required treatment,

\footnotetext{
${ }^{1}$ Department of Psychology and Sports Sciences, University of Hertfordshire, UK

${ }^{2}$ Centre for Health Services and Clinical Services, University of Hertfordshire, UK

${ }^{3}$ Research Department of Clinical, Educational and Health Psychology, University College London, UK

${ }^{4}$ Child Attachment and Psychological Therapies Research Unit (ChAPTRe), Anna Freud National Centre for Children and Families.
} 
Total Difficulties Scores (TDS) from single-informant SDQs submitted at referral were compared to treatment recommendations following routine CAMHS assessment in a realworld setting. To explain the results, clinicians $(n=9)$ from the team were interviewed and key themes identified using Thematic Analysis.

Results: AUROC analysis found that the single informant SDQ discriminated between children who were assessed as needing a mental health intervention and those who did not with low accuracy when SDQs were completed by carers or young people themselves, and moderate accuracy for teacher-completed SDQs. Optimal cut-off scores are calculated and are lower than advised in scoring guidance. Key themes from clinician interviews identified possible gaps and limitations: Developmental Trauma and Attachment Difficulties, A different kind of 'patient?', Seeing the bad but neglecting the sad, and The importance of clinical judgement.

Conclusions: Contrary to current UK Government policy, this study suggests that the single-report SDQ should not be relied upon as a sole means of identifying mental health difficulties in this vulnerable, high-risk population. 


\section{Introduction}

Looked After Children (LAC), or children in 'out-of-home care', are at greater risk of developing mental health difficulties. Studies from North America, Europe and Australasia indicate that around half have clinically significant difficulties, and a further quarter have borderline difficulties (Tarren-Sweeney, 2010). In Britain, Ford, Vostanis, Meltzer \& Goodman (2007) found that 46.4 percent meet the criteria for at least one psychiatric diagnosis, compared to 14.6 percent of children from disadvantaged private households and 8.5 percent from other private households.

In England and Wales, LAC mental health is not routinely assessed by mental health professionals, despite the increased risk in this population. Instead, the children's carers are required to complete the Strengths \& Difficulties Questionnaire (SDQ; Goodman, 1997). The SDQ is a 25 item questionnaire, with scales for emotional disorders, conduct disorders, hyperactivity-inattention, peer problems, prosocial behaviours and total difficulties, plus an optional impact supplement. Closely similar versions for parents, teachers and young people (aged 11-17) can be used individually or combined using a multi-informant algorithm. In a recent meta-analysis, Lavigne, Meyers \& Feldman (2016) identified 19 studies of the parent-report SDQ's classification accuracy in general community and clinical populations. At Goodman's (2001) recommended Total Difficulties Score cut-off of $\geq 17$, they reported a mean sensitivity of .64 and mean specificity of .80 (weighted mean sensitivity $(\mathrm{n}=6) .53$, weighted mean specificity (n=6) .91). Cut-off scores used across studies varied from 10 to 19 . 
The SDQ multi-informant algorithm has been validated as a screening tool for looked after children in Britain. Goodman, Ford, Corbin \& Meltzer (2004) found that when data from multiple informants was combined, the SDQ multi-informant algorithm identified looked after children who met the criteria for any psychiatric diagnosis with a sensitivity of .85 and specificity .80 . However, in LAC mental health screening in England and Wales, only a single-report SDQ-P, completed by the child's carer, is routinely used; additional SDQs are only collected from teachers and young people themselves if a possible difficulty is indicated (HM Government, 2015).

Table 1 provides a summary of studies examining the utility of the single-report SDQ with LAC populations. Goodman et al. (2004) reported insufficient information on the screening efficiency of single-informant SDQs in this population, but indicated that large numbers of children with mental health difficulties would be missed when only a single SDQ was used at the Probable cut-off. Only two studies report both sensitivity and specificity, and only one reports this for a range of cut-off scores. In a large sample of Norwegian foster children aged 6-12, Lehmann et al. (2014) found that a Total Difficulties Score of 13 was optimal (sensitivity $=.83$, specificity .74) for the SDQ-P, completed by the child's carer. Scores of $0-13$ are classified as in the "close to average" range on the SDQ-P (Youth in Mind, 2016). Milburn et al. (2008) also selected a lower score, at the "slightly raised" cut-off point, for their study. Lehmann et al. (2014) highlighted the prevalence of mental health difficulties in children with very low SDQ scores, which ranged between 13 and 29 percent. 
All of the studies in Table 1 define LAC mental health difficulties in terms of whether or not children are found to meet the criteria for one or more psychiatric diagnosis. However, it has been argued that some of the complex mental health difficulties experienced by children with chronic maltreatment histories are not adequately described by the current psychiatric classification system (e.g. van der Kolk, 2005). Clinicians working in specialist services have described LAC with complex difficulties who do not meet the criteria for psychiatric diagnosis but nevertheless require CAMHS intervention (DeJong, 2010). Therefore, the ability of the SDQ to identify LAC who meet the criteria for psychiatric diagnosis may not correspond with its ability to identify LAC who require treatment by specialist CAMHS. Ratnayake et al. (2014) found that only 54 percent of LAC referred to and accepted by a specialist CAMHS had SDQ scores in the clinical range, while for Callaghan et al. (2004) the figure was 78 percent. 
SDQ LAC MENTAL HEALTH

Table 1: Single-informant SDQ sensitivity and specificity values, from a systematic review of the literature (Wright, 2018).

\begin{tabular}{|c|c|c|c|c|c|c|c|}
\hline Study & Sample & Ages & Informant & Criterion & Cut-off score & Sens. & Spec. \\
\hline Goodman & British LAC, & $5-10$ & Carer & DAWBA & Probable* & .51 & - \\
\hline \multirow{4}{*}{2004} & $n=268$ & & Teacher & DAWBA & Probable* & .60 & - \\
\hline & British LAC, & $11-15$ & Carer & DAWBA & Probable* $^{*}$ & .60 & - \\
\hline & $n=271$ & & Teacher & DAWBA & Probable* & .59 & - \\
\hline & & & Self & DAWBA & Probable* & .16 & - \\
\hline Lehmann & Norwegian LAC, & $6-12$ & Carer & DAWBA & TDS 7 & .95 & .32 \\
\hline \multirow[t]{23}{*}{2014} & $n=223$ & & & & TDS 8 & .94 & .36 \\
\hline & & & & & TDS 9 & .91 & .44 \\
\hline & & & & & TDS 10 & .88 & .51 \\
\hline & & & & & TDS 11 & .88 & .61 \\
\hline & & & & & TDS 12 & .86 & .71 \\
\hline & & & & & TDS 13 & .83 & .74 \\
\hline & & & & & TDS 14 & .81 & .75 \\
\hline & & & & & TDS 15 & .77 & .76 \\
\hline & & & & & Impact 1 & .88 & .57 \\
\hline & & & & & Impact 2 & .80 & .70 \\
\hline & & & & & Impact 3 & .65 & .82 \\
\hline & Norwegian LAC, & $6-12$ & Teacher & DAWBA & TDS 7 & .95 & .39 \\
\hline & $\mathrm{n}=195$ & & & & TDS 8 & .95 & .46 \\
\hline & & & & & TDS 9 & .95 & .48 \\
\hline & & & & & TDS 10 & .92 & .50 \\
\hline & & & & & TDS 11 & .88 & .55 \\
\hline & & & & & TDS 12 & .86 & .71 \\
\hline & & & & & TDS 13 & .86 & .77 \\
\hline & & & & & TDS 14 & .83 & .80 \\
\hline & & & & & TDS 15 & .80 & .80 \\
\hline & & & & & Impact 1 & .78 & .67 \\
\hline & & & & & Impact 2 & .65 & .74 \\
\hline & & & & & Impact 3 & .54 & .85 \\
\hline \multirow[t]{2}{*}{ Jee 2011} & USA LAC, $n=45$ & $11-17$ & Carer & ChIPS & $\begin{array}{l}\text { Clinical score on any } \\
\text { subscale or TDS }\end{array}$ & .71 & - \\
\hline & & & Self & ChIPS & $\begin{array}{l}\text { Clinical score on any } \\
\text { subscale or TDS }\end{array}$ & .54 & - \\
\hline \multirow[t]{3}{*}{$\begin{array}{l}\text { Milburn } \\
2008\end{array}$} & $\begin{array}{l}\text { Australian LAC at } \\
\text { entry } n=57\end{array}$ & $4-17$ & $\begin{array}{l}\text { Parents/ } \\
\text { Carers }\end{array}$ & Clinical assessment & Borderline & .80 & .58 \\
\hline & $\begin{array}{l}\text { Australian LAC at } \\
\text { entry } n=32\end{array}$ & & Teacher & Clinical assessment & Borderline & .33 & .50 \\
\hline & $\begin{array}{l}\text { Australian LAC at } \\
\text { entry } n=42\end{array}$ & & Self & Clinical assessment & Borderline & .61 & .92 \\
\hline
\end{tabular}

TDS = total difficulties score. DAWBA = Development And Wellbeing Assessment (Goodman et al., 2000).

ChIPS = Children's Interview for Psychiatric Syndromes (Weller et al., 2000).

* "Probable" prediction derived from priori algorithm on the basis that the relevant symptom score was above the 95th centile and the impact score was two or more. 
The effectiveness of the SDQ as the only method of screening for mental health difficulties in this high-risk population has recently been questioned (House of Commons Education Committee, 2016; SCIE, 2017). Of particular concern is that some targeted CAMHS use the SDQ to set minimum thresholds for acceptance to services, or to inform decisions about who should be offered treatment. For example, in one study, social workers reported that a TDS of 17 was required to access CAMHS in some areas (Cocker, 2016), which would fall in the "high" range on the SDQ-P and SDQ-T or the "slightly raised range on the SDQ-S (Youth in Mind, 2016). If the SDQ is not highly sensitive to mental health difficulties in this population, LAC may be wrongly excluded from accessing CAMHS treatment. Further research in a real-world setting is required and, to this end, the present study examines the utility of the single-report SDQ in referrals to a Targeted CAMHS team in the UK.

\section{Methods}

\section{Design}

This study was developed as part of two-arm, parallel group, single-centre feasibility randomised control trial (RCT) of Mentalization-Based Treatment for children in foster care (MBT-F) (Midgley et al., 2017). The current study had a mixed-methods sequential explanatory design. This comprised an initial quantitative data collection and analysis, which assessed the ability of the single-report SDQ to correctly classify LAC for whom intervention is recommended following routine assessments of referrals to a Targeted CAMHS Team. A second, qualitative phase aimed to explore the reasons why 
some children's mental health difficulties were not identified on referral by the SDQ, from the perspective of clinicians.

\section{Setting}

The study was conducted in a CAMHS Targeted Team for children aged 0-18 who were looked after, adopted or on the edge of care, within a single NHS Foundation Trust in England. This "Targeted Team" is designed for children who do not meet the threshold for mainstream specialist CAMHS, but may have complex difficulties related to experiences of abuse and neglect. The Targeted Team offers consultations to professionals, assessments, brief interventions (comprising 6-12 sessions), and groups for parents and carers. No psychiatry was available in the team, so children requiring psychiatric input were transferred to mainstream CAMHS. Referral criteria stated that, in order to be eligible for treatment, children required a score of 15 or more on the Strengths and Difficulties Questionnaire (SDQ); however, during RCT recruitment, researchers observed that a significant number of children with lower SDQ scores were being accepted for treatment.

\section{Participants}

\section{Phase 1: quantitative sample.}

Of 314 referrals received by the Targeted Team during the recruitment period, 189 were classified as Looked After. (The remainder were on the edge of care or adopted.) Of those, 13 were duplicates, and 32 did not meet the inclusion criteria, leaving a sample of 144 LAC. The available data indicated an even gender split. The largest age group was $11-15$ (36\%); 24\% were aged 8-10, 15\% 4-7, and 14\% 16-17 (no 
SDQ LAC MENTAL HEALTH

age was recorded for $10 \%$ ). Some demographic data was missing because researchers from the wider study had not always recorded this if children were not eligible for the trial.

\section{Phase 2: qualitative sample.}

Nine (of 11 eligible) clinicians agreed to participate in interviews; six were still employed by the team and three had moved to other CAMHS teams. The interview group comprised the clinical team lead, two clinical psychologists, a systemic therapist, three clinical social workers, one psychiatric nurse, and one art therapist. As part of the wider study, approximately half of the clinicians working in the team received training in a specially adapted model of MBT (Midgley et al., 2017).

\section{Measures}

\section{Strengths \& Difficulties Questionnaire.}

The Strengths and Difficulties Questionnaire (Goodman, 1999) is a brief psychiatric screening questionnaire for children aged 3-17. Closely similar versions are available for completion by parents (or carers) (SDQ-P), teachers (SDQ-T), and young people (aged 11-17) (SDQ-S). Any version of the SDQ was accepted by the Targeted Team.

\section{Routine clinical assessment.}

All referrals were assessed by the Targeted Team in line with their usual clinical assessment procedures. Referral information and SDQ scores were examined, and a consultation meeting with the child's professional network was held, in order to gain a better understanding of concerns. Assessments then varied according to the age of the 
child and the nature of the concerns. Typically, assessments comprised clinical interviews with the carer and/or child, observation of carer and child together, and contact with the child's school. The case was then discussed at the weekly multidisciplinary team meeting and a decision as to whether or not to offer treatment was agreed.

\section{Procedure}

The wider study was reviewed by the East of England - Cambridge NRES Committee (REC reference: $15 / \mathrm{EE} / 0332$ ) and given a favourable ethical opinion on $29^{\text {th }}$ December 2015. The present study was an amendment to the ethics application for the wider study, and was approved on $17^{\text {th }}$ January 2018 .

All referrals submitted to the Targeted Team between 2 January 2016 and 14 July 2017 were screened for inclusion in the present study. Participants were eligible if they were: aged 4-17; had been Looked After (in foster, kinship or residential care) for 4 weeks or more; had one or more SDQs submitted with the referral; and had a referral outcome recorded within the data collection period. Details of referrals were recorded in the Recruitment Log, including the child's looked after status, SDQ score and the outcome of the referral following routine assessment by the multi-disciplinary. SDQs were not administered by the research team; they were submitted with referrals, so no administration protocol was followed. SDQs were hand-scored using guidance from Youth in Mind (2016), in line with usual practice in the Targeted Team. Referral outcomes were coded as follows: 1. CAMHS treatment recommended (this included 


\section{SDQ LAC MENTAL HEALTH}

offers of treatment by the Targeted Team or referrals to other CAMHS teams locally, or further afield if children were moving out of area) 2. Other mental health treatment or neurodevelopmental assessment recommended (this included counselling, play or art therapy at school, specialist voluntary sector services for survivors of sexual abuse and traumatised refugees, a local authority-led service for attachment and trauma difficulties, as well as referrals to education psychology services) 3 . No treatment recommended. If treatment was recommended but deferred, for example until court proceedings had finished or a placement move had been completed, it was still recorded as recommended. Receiver Operating Characteristics (ROC) analyses were conducted on the Total Difficulties Scores (TDS) and referral outcomes, using easyROC version 0.3. Separate Area Under the Receiver Operating Characteristics (AUROC) values were estimated for the scores reported by caregivers, teachers and self-report questionnaires, and optimal cut-off values identified. SPSS version 24 for Macintosh was used for additional statistical analyses.

In the second, qualitative phase, semi-structured interviews were conducted with clinicians from the Targeted Team to investigate why some children were offered treatment despite having SDQ total difficulties scores in the "close to average" or "slightly raised" range (Youth in Mind, 2016; or see Tables 3-5 below for a summary of threshold for each band, by informant type). All clinicians employed by the Targeted Team during the course of the feasibility RCT $(n=11)$ were invited to participate in semistructured interviews. Clinicians were asked about the work of the Targeted Team, clinicians' role within the team, how referrals and assessments were conducted, and the 
role of the SDQ in this. Reasons for offering a mental health intervention to children for whom the SDQ indicated a relatively low level of need were explored. Finally, clinicians were asked about their views about the utility of the SDQ within the service.

Interviews were recorded and transcribed, and Thematic Analysis was employed to identify key themes, using the steps outlined by Joffe (2012). These were: examining the full dataset and developing a coding frame; checking the reliability of the coding frame by comparing coding from two independent coders across 10 percent of the dataset; coding the full dataset in NVivo; and conducting the analysis by identifying patterns, meanings and connections in the codes in order to construct a map of key themes and subthemes.

\section{Results}

\section{Strengths \& Difficulties Questionnaires}

The service requirement was that at least one SDQ should be provided with referrals. 179 SDQs for 144 children were collected from referrals; 113 children had 1 SDQ, 27 children had 2 SDQs and 4 had 3 SDQs. This suggests that social workers were not routinely collecting SDQs from multiple informants. Of the available SDQs, 97 were parent/carer-report versions (SDQ-P), 41 were teacher-report versions (SDQ-T) and 41 self-report versions (SDQ-S). Mean Total Difficulties Scores (TDS) at referral, by respondent type, were as follows: carer-report $\mathrm{SDQ}, M=18.02, S D=7.38$; teacher-report $\mathrm{SDQ}, M=17.20, S D=7.24$, self-report SDQ, $M=15.10, S D=6.67$. The Targeted Team 
is commissioned to work with children who score 15 or more on the SDQ; however, 65 (36\%) of the SDQs submitted with referrals had a TDS of less than 15.

\section{Treatment Recommendations}

Of the 144 children referred to the service with at least 1 SDQ, $95(66 \%)$ were offered a service from the Targeted Team or referred to another CAMHS service. For a further $21(14.6 \%)$ children, other types of mental health or neurodevelopmental services were recommended. In total, $80.6 \%(n=116)$ of children referred to the service were assessed to be in need of some form of intervention to support their mental health or neurodevelopment. No treatment was deemed necessary for 28 (19.4\%) children.

\section{Accuracy of the SDQ in identifying assessed mental health needs}

Table 2: Area Under the ROC Curve (AUROC) for the SDQ, by respondent.

\begin{tabular}{lllllcc}
\hline & \multicolumn{2}{l}{ SDQ-P $(\mathbf{n = 9 7})$} & \multicolumn{2}{l}{ SDQ-T $(\mathbf{n = 4 1 )}$} & \multicolumn{2}{l}{ SDQ-S (n=41) } \\
\hline & \multicolumn{2}{l}{ Treatment recommendation } & \multicolumn{2}{l}{ Treatment recommendation } & \multicolumn{2}{c}{ Treatment recommendation } \\
& CAMHS & Any MH & CAMHS & Any MH & CAMHS & Any MH \\
\hline Area Under the ROC curve & 0.620 & 0.613 & 0.810 & 0.735 & 0.568 & 0.565 \\
Standard error & 0.061 & 0.071 & 0.068 & 0.084 & 0.112 & 0.157 \\
$95 \%$ Confidence interval & $0.501-0.739$ & $0.473-0.753$ & $0.677-0.943$ & $0.571-0.899$ & $0.348-0.787$ & $0.258-0.872$ \\
z statistic & 1.978 & 1.578 & 4.565 & 2.805 & 0.603 & 0.416 \\
Significance level P (Area=0.5) & 0.048 & 0.114 & $<.001$ & 0.005 & 0.547 & 0.678 \\
\hline
\end{tabular}

Estimation method is DeLong (1988).

The Area Under the Receiver Operating Curve (AUROC) is used to assess the accuracy of the SDQ in identifying assessed mental health need (as defined in the method section) in CAMHS-referred LAC. AUROC analyses indicated that the SDQ Total Difficulties Scores (TDS) predicted recommendations of mental health treatment better 
than chance for questionnaires completed by carers (SDQ-P) and teachers (SDQ-S). The ability of the SDQ-P to discriminate between children who were offered treatment by the Targeted Team or another CAMHS team (.62) or recommended any mental health treatment (.61), and those who do not require treatment, was in the low range (according to criteria described by Streiner \& Cairney, 2007). For the smaller group of children for whom an SDQ-T was provided $(n=41)$, discrimination was better; it distinguished between those children who were recommended CAMHS treatment (.81) or any mental health treatment (.74) and those who were not, with accuracy that fell within the "moderate" range (as per Streiner \& Cairney, 2007). The SDQ-S, completed by young people (aged 11+) themselves, failed to reliably identify children who were recommended treatment from CAMHS or any mental health treatment, performing no better than chance $(\mathrm{p}>.05)$.

\section{Optimal cut-off scores}

For each informant type, ROC analysis was used to select the optimal cut-off point for the SDQ to discriminate between children who were recommended treatment from CAMHS, or any mental health treatment, and those who did not require treatment (see Tables 3, 4 and 5, below). Optimal cut-off scores were calculated using two methods: first, by giving equal weight to sensitivity and specificity (as per Youden's Index); and second, by prioritizing sensitivity that is $\geq .85$. Glascoe (2005) recommends that sensitivity and specificity of $>80$ is desirable in screening where sensitivity and specificity are equally important. It is our view that in this high-risk group, the social, ethical and financial costs of failure to identify and treat mental health difficulties in a 
timely way (e.g. Minnis, 2006) is greater than the cost of completing further assessment of children who are found not to require treatment, and therefore sensitivity should be prioritised. To achieve sensitivity $\geq .85$, the optimal Total Difficulties Score cut-off value for predicting CAMHS treatment were as follows: SDQ-P TDS=12 (sensitivity $=.85$, specificity $=.30)$, SDQ-T TDS $=13($ sensitivity $=.88$, specificity $=.47)$ and SDQ-S $\mathrm{TDS}=8$ (sensitivity $=.86$, specificity $=.43$ ). These scores would usually be classified in the "close to average" range for the SDQ-P and SDQ-S, and the "slightly raised" range for the SDQ-T, and there would be high rates of false positives, while as many as 3 in 20 children with mental health difficulties would still be missed. Optimal cut-off scores for predicting any type of mental health treatment were lower: SDQ-P TDS=10 (sensitivity $=$ .87 , specificity $=.20)$, SDQ-T TDS $=10($ sensitivity $=.90$, specificity $=.36)$, SDQ-S $\mathrm{TDS}=8($ sensitivity $=.88$, specificity $=.43)$. 
Table 3: Receiver Operating Characteristics Analyses for the SDQ-P Total Difficulties

Scale from the parent/carer sample $(\mathrm{n}=97)$

\begin{tabular}{|c|c|c|c|c|c|c|c|c|c|c|c|c|c|c|c|}
\hline \multicolumn{8}{|c|}{ CAMHS treatment } & \multicolumn{8}{|c|}{ Any MH treatment } \\
\hline TDS & Sens & $95 \% \mathrm{Cl}$ & Spec & $95 \% \mathrm{Cl}$ & $P P V$ & $N P V$ & $+L R$ & $T D S$ & Sens & $95 \% \mathrm{Cl}$ & Spec & $95 \% \mathrm{Cl}$ & $P P V$ & $N P V$ & $+L R$ \\
\hline 9 & .97 & $.89,1$ & .16 & $.06, .32$ & .65 & .75 & 1.15 & 9 & .94 & $.86, .98$ & .15 & $.03, .38$ & .81 & .38 & 1.10 \\
\hline 10 & .90 & $.80, .96$ & .22 & $.10, .38$ & .65 & .57 & 1.15 & $10^{* *}$ & .87 & $.77, .94$ & .20 & $.06, .44$ & .81 & .29 & 1.09 \\
\hline 11 & .88 & $.77, .95$ & .27 & $.14, .44$ & .66 & .59 & 1.21 & 11 & .84 & $.74, .92$ & .25 & $.09, .49$ & .81 & .29 & 1.13 \\
\hline $12^{* *}$ & .85 & $.73, .93$ & .30 & $.16, .47$ & .66 & .55 & 1.21 & 12 & .82 & $.71, .90$ & .30 & $.12, .54$ & .82 & .30 & 1.17 \\
\hline 13 & .82 & $.70, .91$ & .35 & $.20, .53$ & .67 & .54 & 1.26 & 13 & .78 & $.67, .87$ & .35 & $.15, .59$ & .82 & .29 & 1.20 \\
\hline 14 & & & & & & & & 14 & & & & & & & \\
\hline 15 & .75 & $.62, .85$ & .46 & $.30, .63$ & .70 & .53 & 1.39 & 15 & .70 & $.59, .80$ & .45 & $.23, .69$ & .83 & .28 & 1.28 \\
\hline 16 & & & & & & & & 16 & & & & & & & \\
\hline $17^{*}$ & .67 & $.53, .78$ & .57 & $.40, .73$ & .71 & .51 & 1.54 & 17 & .62 & $.51, .73$ & .60 & $.36, .81$ & .86 & .29 & 1.56 \\
\hline 18 & .60 & $.47, .72$ & .59 & $.42, .75$ & .71 & .48 & 1.48 & 18 & .57 & $.45, .68$ & .65 & $.41, .85$ & .86 & .28 & 1.63 \\
\hline 19 & .53 & $.40, .66$ & .62 & $.45, .78$ & .70 & .45 & 1.41 & 19 & & & & & & & \\
\hline 20 & .50 & $.37, .63$ & .68 & $.50, .82$ & .71 & .46 & 1.54 & $20^{* *}$ & .48 & $.37, .60$ & .70 & $.51, .91$ & .88 & .27 & 1.92 \\
\hline
\end{tabular}

${ }^{*}$ Optimal cut-off score if giving equal weight to sensitivity and specificity (Youden's Index). ${ }^{* *}$ Optimal cut-off score if

sensitivity $\geq .85$. N.B. according to the hand-scoring guidance available on the official sdq.info website, SDQ-P scores

$\leq 13$ are in the "close to average" range, 14-16 are "slightly raised", 17-19 are "high" and $\geq 20$ are "very high".

Table 4: Receiver Operating Characteristics Analyses for the SDQ-T Total Difficulties

Scale from the teacher sample $(\mathrm{n}=41)$.

\begin{tabular}{|c|c|c|c|c|c|c|c|c|c|c|c|c|c|c|c|}
\hline \multicolumn{8}{|c|}{ CAMHS treatment } & \multicolumn{8}{|c|}{ Any MH treatment } \\
\hline TDS & Sens & $95 \% \mathrm{Cl}$ & Spec & $95 \% \mathrm{Cl}$ & $P P V$ & $N P V$ & $+L R$ & TDS & Sens & $95 \% \mathrm{Cl}$ & Spec & $95 \% \mathrm{Cl}$ & $P P V$ & $N P V$ & $+L R$ \\
\hline 9 & & & & & & & & 9 & .93 & $.78, .99$ & .18 & $.02, .52$ & .76 & .50 & 1.14 \\
\hline 10 & .96 & $.79,1.0$ & .35 & $.14, .62$ & .68 & .86 & 1.48 & $10^{* *}$ & .90 & $.74, .98$ & .36 & $.11, .69$ & .79 & .57 & 1.41 \\
\hline 11 & & & & & & & & 11 & & & & & & & \\
\hline 12 & .92 & $.73, .99$ & .47 & $.23, .72$ & .71 & .80 & 1.73 & 12 & .83 & $.65, .94$ & .46 & $.17, .77$ & .81 & .50 & 1.53 \\
\hline $13^{* *}$ & .88 & $.68, .97$ & .47 & $.23, .72$ & .70 & .73 & 1.65 & 13 & & & & & & & \\
\hline 14 & & & & & & & & 14 & .77 & $.58, .90$ & .55 & $.23, .83$ & .82 & .46 & 1.69 \\
\hline 15 & .83 & $.63, .95$ & .65 & $.38, .86$ & .77 & .73 & 2.36 & 15 & .73 & $.54, .88$ & .64 & $.31, .89$ & .85 & .47 & 2.02 \\
\hline 16 & & & & & & & & 16 & & & & & & & \\
\hline $17^{*}$ & .79 & $.58, .93$ & .71 & $.44, .90$ & .79 & .71 & 2.69 & 17 & & & & & & & \\
\hline 18 & .71 & $.49, .87$ & .77 & $.50, .93$ & .81 & .65 & 3.01 & 18 & .60 & $.41, .77$ & .73 & $.39, .94$ & .86 & .40 & 2.2 \\
\hline 19 & .67 & $.45, .84$ & .82 & $.57, .96$ & .84 & .64 & 3.78 & $19^{*}$ & .57 & $.37, .75$ & .82 & $.48, .98$ & .90 & .41 & 3.12 \\
\hline 20 & .50 & $.29, .71$ & .88 & $.64, .99$ & .86 & .57 & 4.25 & 20 & .43 & $.26, .63$ & .91 & $.59,1.0$ & .93 & .37 & 4.77 \\
\hline
\end{tabular}

${ }^{*}$ Optimal cut-off score if giving equal weight to sensitivity and specificity (Youden's Index). ${ }^{* *}$ Optimal cut-off score if sensitivity $\geq .85$. N.B. according to the hand-scoring guidance available on the official Youth In Mind website, SDQ-T scores $\leq 11$ are in the "close to average" range, 12-15 are "slightly raised", 16-18 are "high" and $\geq 19$ are "very high". 


\section{SDQ LAC MENTAL HEALTH}

Table 5: Receiver Operating Characteristics Analyses for the SDQ-S Total Difficulties

Scale from the self-report sample.

\begin{tabular}{|c|c|c|c|c|c|c|c|c|c|c|c|c|c|c|c|}
\hline \multicolumn{8}{|c|}{ CAMHS treatment } & \multicolumn{8}{|c|}{ Any MH treatment } \\
\hline TDS & Sens & $95 \% \mathrm{Cl}$ & Spec & $95 \% \mathrm{Cl}$ & $P P V$ & NPV & $+L R$ & $T D S$ & Sens & $95 \% \mathrm{Cl}$ & Spec & $95 \% \mathrm{Cl}$ & $P P V$ & $N P V$ & $+L R$ \\
\hline $8^{\star \star}$ & .86 & $.68, .96$ & .25 & $.06, .57$ & .74 & .43 & 1.15 & $8^{*}, * *$ & .88 & $.73, .97$ & .43 & $.10, .82$ & .88 & .43 & 1.54 \\
\hline 9 & & & & & & & & 9 & & & & & & & \\
\hline 10 & & & & & & & & 10 & & & & & & & \\
\hline 11 & & & & & & & & 11 & & & & & & & \\
\hline 12 & & & & & & & & 12 & & & & & & & \\
\hline $13^{*}$ & .79 & $.60, .92$ & .42 & $.15, .72$ & .77 & .46 & 1.36 & 13 & & & & & & & \\
\hline 14 & & & & & & & & 14 & & & & & & & \\
\hline 15 & .62 & $.42, .79$ & .58 & $.28, .85$ & .78 & .39 & 1.49 & 15 & .59 & $.41, .75$ & .57 & $.18, .90$ & .87 & .22 & 1.37 \\
\hline 16 & & & & & & & & 16 & & & & & & & \\
\hline 17 & .52 & $.33, .71$ & .67 & $.35, .90$ & .79 & .36 & 1.55 & 17 & & & & & & & \\
\hline 18 & .35 & $.18, .54$ & .75 & $.43, .95$ & .77 & .32 & 1.38 & 18 & .32 & $.17, .51$ & .72 & $.29, .96$ & .85 & .18 & 1.32 \\
\hline 19 & & & & & & & & 19 & & & & & & & \\
\hline 20 & & & & & & & & 20 & & & & & & & \\
\hline
\end{tabular}

${ }^{*}$ Optimal cut-off score if giving equal weight to sensitivity and specificity (Youden's Index). ${ }^{* *}$ Optimal cut-off score if sensitivity $\geq .85$. N.B. according to the hand-scoring guidance available on the official Youth In Mind website, SDQ-S scores $\leq 14$ are in the "close to average" range, 15-17 are "slightly raised", 18-19 are "high" and $\geq 20$ are "very high".

\section{Thematic Analysis}

The Thematic Analysis identified four main themes in relation to the use of the SDQ as a screening tool for referrals to the Targeted Team. These were: Developmental Trauma and Attachment Difficulties, A different kind of 'patient?', Seeing the bad but neglecting the sad, and The importance of clinical judgement. A thematic map, indicating how these themes and their subthemes fit together, and the number of participants whose data was coded against each theme, is provided in Figure 1. 

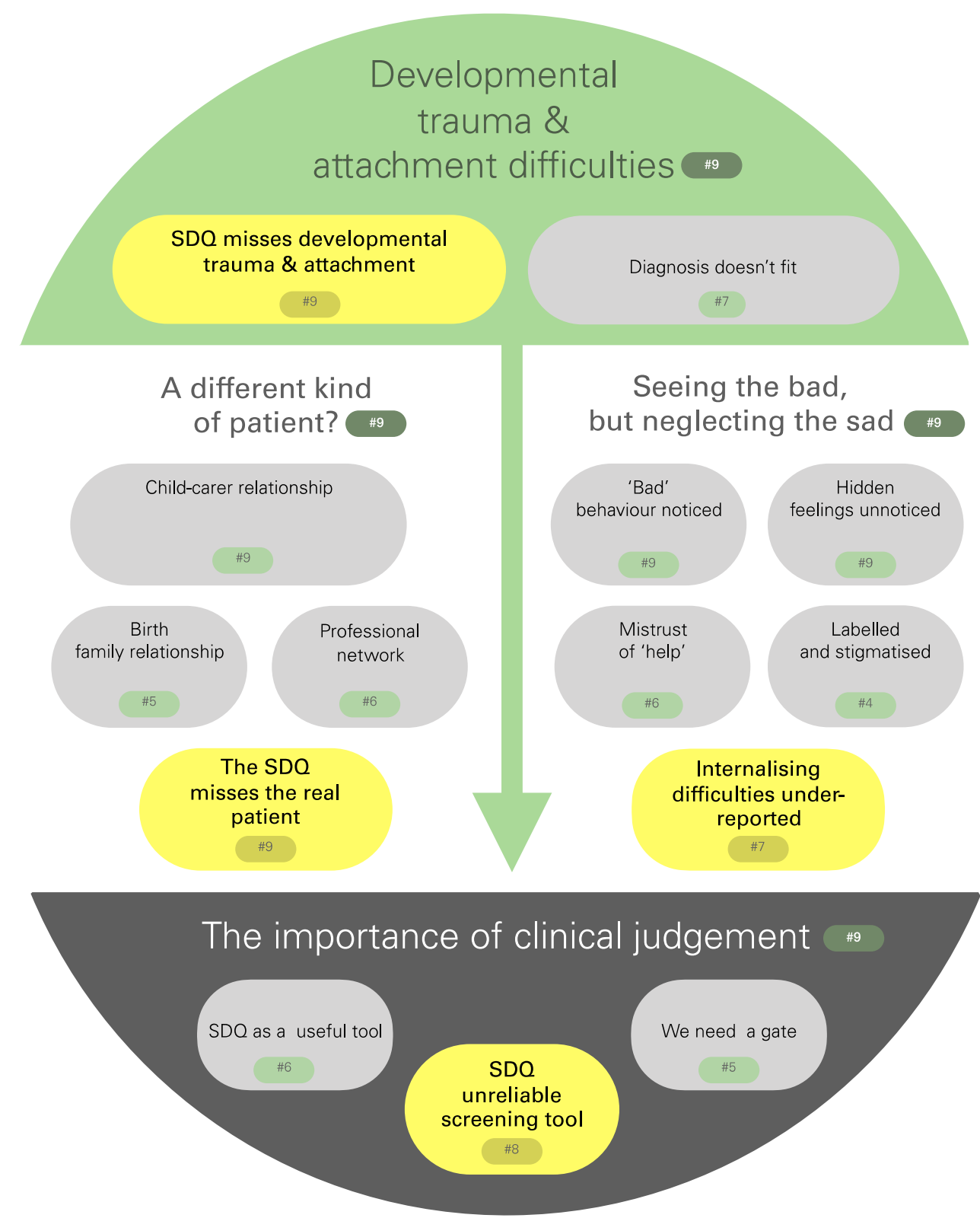

Figure 1: Thematic map showing key themes and sub-themes identified via Thematic Analysis. $\#=$ number of clinicians linked to theme.

Theme 1: Developmental trauma \& attachment difficulties. 
SDQ LAC MENTAL HEALTH

All of the clinicians interviewed linked the types of difficulties experienced by the children they worked with to experiences of interpersonal trauma in their early development. They described patterns of repeated dysregulation (particularly emotional, behavioural and relational), impairment in everyday functioning, and persistently altered expectations (especially distrust of caregivers and professionals from health and social care). A subtheme, Diagnosis doesn't fit, described a perception that children with developmental trauma and attachment difficulties did not tend to fit neatly into the existing diagnostic classification system.

The most frequently cited criticism of the SDQ was that The SDQ misses developmental trauma and attachment difficulties. This was described by almost all $(n=8)$ of the participants. This perception seemed to influence clinicians' views on the suitability of the SDQ for use with the looked after population.

"In the children that I work with or the team works with, I don't have that much faith in them [SDQs] because I don't think they really capture what the children's difficulties are. They don't really bring out the attachment issues and there's not much scope for developmental trauma issues to be brought up either."

Clinician 1

When clinicians reported that the SDQ had under-estimated the severity of difficulties experienced by children referred to the team, complex presentations arising from interpersonal trauma were typically described. 
“...really clear attachment difficulties and not being able to get a kind of different relationship with each carer but emotional outbursts, going into a kind of frozen state, enuresis, and distress and like... all trauma and attachment-related stuff. Complex, very complex so... it is interesting how they sometimes don't score very high when you think of the complexity of the case."

\section{Clinician 7}

Some clinicians suggested that the SDQ was more appropriate for children who had a clearer presentation linked to specific symptoms.

"I think if you're anxious, you're angry, or you're sad, the SDQs will pick it up, okay, but when you got like internalised trauma, or odd ideas or the inability to sort of work effectively, socially, and all that, it doesn't pick it up very well...”

Clinician 2

\section{Theme 2: A different kind of 'patient'.}

When clinicians described the difficulties that they were trying to change through their interventions, they seemed to be describing $A$ different kind of 'patient' - not the children themselves, but the children's relationships with their caregivers. This focus on 
the child in the context of their relationships was sometimes described as at odds with the views of other professionals in the children's lives, who expected clinicians to "fix" the child individually. Most frequently, the child's relationship to their foster carer was seen as the target of the intervention, although sometimes relationships with the wider professional network, or the child's birth family, were prioritised. Clinicians explained that these relationships were influenced by child's complex relational history, which often interfered with their capacity to form positive relationships with those now trying to care for them; and by carers' understanding of and responses to children's expressions of these difficulties.

Many clinicians expressed a view that the SDQ had limited value in working with this client group because it focuses on symptoms within the child and does not capture difficulties in their relationships with their caregivers. As such, an important sub-theme was: The $S D Q$ misses the real "patient".

"The SDQ doesn't include items that focus on how children relate to other people... So, some of the difficulties that specifically stem from adverse childhood experiences that were really impacting on children's placements, so children being very controlling, trying to keep control and being very adult in their presentation, not being able to kind of tolerate boundaries from adults... Those kinds of things are not well-captured on the SDQ... but were underpinning quite a lot of the instability in placements and things that we were seeing."

Clinician 8 
Because the SDQ does not assess children in the context of their caregiving relationships, clinicians expressed concern that SDQ scores might not fully capture the difficulties that were being targeted, both at assessment and at follow-up if used as an outcome measure. Changes in the quality of the relationship between carer and child were viewed as a valuable outcome, even where children's presentations had not changed significantly over the course of the intervention.

"I think sometimes when people look at the difference in scores, they might not see a lot - and that's what commissioners look at - but actually, there's been a lot of positive changes... the placement hasn't broken down because actually the carers have got a bigger understanding and they can then change or adapt how they might approach the child because of that understanding."

\section{Clinician 9}

\section{Theme 3: Seeing the "bad" and neglecting the sad.}

Clinicians reported that the children they worked with were usually referred to the service as a result of behaviour that was causing problems for their carers, schools and others. "Bad behaviour" was noticed and responded to by the professional network, to the extent that some clinicians described difficulty in seeing the children beneath the numerous pathologising labels that they had accrued. Meanwhile, hidden feelings were more likely to go unnoticed by the adults in the child's life, especially those that did not cause overt disruption at home or school. 
SDQ LAC MENTAL HEALTH

Almost all clinicians $(n=8)$ described or gave an example of the SDQ missing less 'noisy' difficulties in children. SDQs from carers and teachers were typically described as more likely to report difficulties with behaviour, and less likely to identify emotional difficulties.

"So this child who's in the fight or flight mode is sort of really acting out and would really probably show up on an SDQ because it'll be like all there - everything's externalised - whereas a child who's actually quite withdrawn and compliant, they might not really come up on that but actually there's a lot to be a bit worried about with them..."

\section{Clinician 7}

One of the reasons why interviewees thought that carers and teachers might not pick up on children's emotional difficulties might be the children's mistrust of help. Most clinicians $(n=7)$ stated that they considered the possibility of under-reporting by young people themselves when reviewing the SDQ-S. This may explain the particularly poor performance of the SDQ-S in this study.

“...is the child underreporting certain concerns or over reporting strengths? For various reasons... it might be, you know, just wanted to be seen as normal or just want to please the parent or the carer, which is a common dynamic in looked after children, um, or just not knowing whether... reporting it honestly might lead to something bad for the child."

Clinician 3 
As a result of initial under-reporting, some clinicians described how an increase in self-reported SDQ scores over the course of therapy might actually represent positive progress in therapy. This was because clinicians felt that young people might be more willing to report their symptoms honestly as the therapeutic relationship developed.

\section{Theme 4: The importance of clinical judgement.}

Given the complexity of the difficulties affecting children and their care networks, clinicians described The importance of clinical judgement when making decisions about whether treatment should be offered. Although the majority of clinicians $(n=6)$ felt that the SDQ could be $A$ useful tool as part of a wider assessment, all of the clinicians interviewed reported using clinical judgement to over-ride the service requirement for an SDQ score of 15 or more, if other evidence suggested that it might be under-estimating the level of difficulty.

“Occasionally we will accept them if they're lower [on the SDQ] because actually, the information written [on the referral] really very clearly shows that this child or, you know, this family is in distress and there's clearly a mental health component. It doesn't show on the SDQ, but it's very clear so... so they don't match up, but actually, the risk factors are enough... that we'll respond."

Clinician 6 
A subtheme, the $S D Q$ as an unreliable screening tool for this population, seemed to be another reason why clinicians viewed clinical judgement as so important in their work. Comprehensive assessment, including accounts from multiple sources in the child's life, was deemed particularly important because of the complexity of the difficulties experienced by children, their carers and networks.

"[The SDQ] gives us a sense that we sort of know what we're doing... but it's a false sense I think, because mostly we don't, and that's fair enough. We don't and we probably shouldn't because it's complicated and difficult, and it should be. These things [SDQs] try to reduce, don't they? They're reductive. They try to reduce the complexity of the things that we're doing because it's too much for us... so it's an attempt in a way to reduce the anxieties that we experience about the work that we do..."

\section{Clinician 4}

Despite these limitations, clinicians reported that the SDQ played an important role as a gatekeeper to prevent the service from being overwhelmed with referrals.

"I wouldn't have put a huge amount of weight on the score alone, but I think as a team, we try to because we had to have this gate. There had to be a line somewhere in terms of managing referrals."

\section{Clinician 8}

However, there was also a suggestion that the use of the SDQ as a means of gatekeeping access to services could be misused. Carers and professionals might overreport on the SDQ in order to gain access to the service. 
SDQ LAC MENTAL HEALTH

"It kind of makes me suspicious that the carers and teachers would maybe... sometimes overly sort of describe things, especially the things that they look at and think are the key things that might get service..."

\section{Clinician 5}

Three clinicians described a sense of pressure to stick to the service requirement of a score of 15 or above on the SDQ in a context of limited capacity and high demand. Concerns were expressed that some children with low SDQ scores who needed a service might miss out because of this. However, efforts to ensure that this did not happen, such as holding consultations and drop-in clinics, were described.

"I think that the problem is people who are non-clinical see them as, er, a sort of gospel. "Oh, this says that, therefore it must be so." It's dangerous, and it's dangerous in a sense that operational managers, because they are not clinically based, will think no, it's 15 or bust, and yet the evidence is that actually there's quite a serious problem going on, and although they scored under 15 , you've still got to see them."

Clinician 2

\section{Discussion}

This study aimed to further understanding of the utility of the single-report SDQ as a screening measure for mental health difficulties in looked after children referred to a CAMHS Targeted Team in England. We found that the accuracy of the single-informant SDQ in distinguishing between children who were assessed as needing mental health treatment, and those who did not, was low when the SDQ-P was completed by carers, and 


\section{SDQ LAC MENTAL HEALTH}

was not statistically different from chance when the SDQ-S was completed by young people themselves. SDQs from these informants only achieved sensitivity of $>=.85$ if the cut-off scores used were much lower than official guidance suggests, resulting a in high proportion of false positives. The SDQ-T performed considerably better, with accuracy in the moderate range. It is important to note that only 29 percent of children in the study had an SDQ-T, and it may be that teachers are more inclined to complete SDQ-Ts for children who display the types of externalising difficulties that have been found to be more accurately captured by the SDQ, such as hyperkinetic disorder and ADHD (Goodman et al., 2004).

Qualitative data from clinician interviews provided some insight into why the single-report SDQ failed to identify some children's mental health difficulties. First, the SDQ was designed to detect common mental health diagnoses and not complex difficulties arising from developmental trauma, which are common in this population and may be inadequately described by existing diagnostic classifications (DeJong, 2010; van der Kolk, 2005). Second, the SDQ looks for mental health difficulties within children, and is not designed to measure the ways in which early interpersonal trauma, attachment difficulties and additional challenges of the care system are played out in caregiving relationships. Third, the SDQ is less sensitive to emotional difficulties (Goodman et al., 2004); this may be especially true in the LAC population, where stigma and mistrust of professionals may lead to feelings being hidden or masked. Fourth, when assessing the needs of this complex and vulnerable population, clinicians argued that careful clinical 


\section{SDQ LAC MENTAL HEALTH}

judgement is required in order to make decisions about whether children would benefit from input from mental health services.

The study provides empirical support for the SCIE Expert Working Group finding that the SDQ "by itself is not an effective way of measuring the mental health and emotional wellbeing of young people" (SCIE 2017, p.7). Therefore, eligibility criteria for LAC CAMHS services should not exclude looked after children with low SDQ scores from accessing mental health assessment from a suitably qualified clinician, and appropriate treatment, if there are concerns about their mental health. This does not mean that the SDQ has no utility in LAC services, as part of a wider assessment.

Looked after children who hide their emotional distress may be at greater risk of missing out on mental health treatment (Golding, 2010), and the results presented here suggest that relying on the single-informant SDQ as a means of assessing need for specialist services risks compounding this problem. Children who "express emotional distress through passive, withdrawn or compliant behavior" should be equally prioritised by CAMHS (NICE, 2010, Recommendation 8: Commission mental health services, p.35). Golding (2010) suggests that interagency meetings can help to highlight concerns that might otherwise be overlooked. Brief measures designed to identify internalizing difficulties in general populations, such as the Revised Children's Depression and Anxiety Scale (RCADS; Chorpita, 2000), may be helpful as part of a wider assessment. Measures specifically designed for the LAC population, such as the Assessments Checklists for Children (ACC) and Adolescents (ACA) may help to highlight types of 


\section{SDQ LAC MENTAL HEALTH}

internalising difficulties commonly found in children who have experienced developmental trauma, such as insecure or anxious-distrustful behaviours or negative self-image (Tarren-Sweeney, 2018).

Theories of attachment and developmental trauma predict that children who have experienced interpersonal trauma in early life will present particular challenges to their carers. Caring for these children can lead to vicarious trauma, burnout and "blocked" caregiving capacity (Baylin \& Hughes 2010). This will not be captured by the SDQ. In the present study, alternative measures capturing aspects of children's relationships were suggested by clinicians; these included the Parent Reflective Functioning Questionnaire (Luyten, Mayes, Nijssens \& Fonagy, 2017) and the Thinking About Your Child questionnaire (also known as the Carer's Questionnaire; Wassall, Golding, \& Barnbrook, 2011). Carers may find it difficult to admit to difficulties, and clinical judgement will be required when using measures.

It is important to recognise clinicians' fears that, in a service context increasingly characterized by limited capacity and rising demand, removing the SDQ as a "gate" to service could result in a level of demand that they cannot meet. There is no avoiding the fact that better identification of mental health difficulties in looked after children will result in more children requiring intervention for their mental health difficulties, and there are no easy answers to how already stretched services can increase the number of children they support without additional investment. However, this population is at high risk of adverse outcomes such as leaving school without qualifications, using drugs, 
SDQ LAC MENTAL HEALTH

going to prison and becoming homeless, so in terms of health economics, greater investment in services for this vulnerable group is likely to pay dividends in the long term (Silver, Golding \& Roberts, 2015). Active steps must be taken to avoid perpetuating the neglect of traumatised children who do not demand or expect attention by excluding them from access to CAMHS services.

Various limitations of this study should be noted. The study focused on a sample of looked after children referred to a CAMHS service, not all looked after children in the local authority, and the sample is therefore likely to contain a higher level of mental health difficulties than the general LAC population, so the extent to which findings can be generalised is unclear. Given that an SDQ score of 15 is formally required for access to this particular service, it is possible that some social workers did not even make a referral to the Targeted CAMHS Team if the child they were concerned about had scored below 15 on the SDQ, and this may also have impacted on the predictive values found here. This was an opportunity sample: future research should include all LAC in a local authority area. Another limitation is that the assessments of children's mental health needs were not standardized, and were not based solely on diagnostic criteria, because this study took place in a real-world Targeted CAMHS setting. However, given that psychiatric diagnoses may not fully capture the types of mental health difficulties arising from early maltreatment, the outcome of an assessment of the child's referral by a multidisciplinary Targeted CAMHS team is a useful reference standard. 
SDQ LAC MENTAL HEALTH

Overall, the results of this study suggest that the single-report SDQ should not be relied upon to identify LAC who require treatment for their mental health. This has important implications for clinical practice, service design and policy. Clinicians, commissioners and policy-makers should review its role in gatekeeping access to CAMHS for this vulnerable, high-risk population.

\section{References}

Callaghan, J., Young, B. Pace, F. \& Vostanis, P. (2004) Evaluation of a New Mental Health Service for Looked after Children. Clinical Child Psychology and Psychiatry, 9(1) $1359-1045$.

Cocker, C. (2016) A critical evaluation of the usefulness of the SDQ in social workers' assessments of looked after children's mental health.

https://www.uea.ac.uk/documents/3437903/12381690/Christine+Cocker.pdf/6bde8ce0$\underline{\text { acb5-4ebf-98a7-ae46e86c5153 }}$

DeJong, M. (2010). Some reflections on the use of psychiatric diagnosis in the looked after or "in care" child population. Clinical Child Psychology and Psychiatry, 15(4), 589599.

Ford, T., Vostanis, P., Meltzer, H., \& Goodman, R. (2007). Psychiatric disorder among British children looked after by local authorities: comparison with children living in private households. The British Journal of Psychiatry, 190(4), 319-325. 
Glascoe, F. P. (2005). Screening for developmental and behavioral problems. Mental retardation and developmental disabilities research reviews, 11(3), 173-179.

Golding, K.S. (2010) Multi-agency and specialist working to meet the mental health needs of children in care and adopted. Clinical Child Psychology and Psychiatry, 15(4), $573-587$.

Goodman, R. (1997). The Strengths and Difficulties Questionnaire: a research note. Journal of child psychology and psychiatry, 38(5), 581-586.

Goodman, R., Ford, T., Corbin, T., \& Meltzer, H. (2004). Using the Strengths and Difficulties Questionnaire (SDQ) multi-informant algorithm to screen looked-after children for psychiatric disorders. European Child \& Adolescent Psychiatry, 13(2), 2531.

HM Government (2015) Promoting the health and well-being of looked-after children: Statutory guidance for local authorities, clinical commissioning groups and NHS England. London: TSO.

House of Commons Education Committee (2016) Mental health and well-being of looked-after children. Fourth Report of Session 2015-16. 
Jee, S. H., Szilagyi, M., Conn, A. M., Nilsen, W., Toth, S., Baldwin, C. D., \& Szilagyi, P. G. (2011). Validating office-based screening for psychosocial strengths and difficulties among youths in foster care. Pediatrics, 127(5), 904-910.

Joffe, H. (2012) Thematic analysis. In D. Harper \& A.R. Thompson (Eds) Qualitative research methods in mental health and psychotherapy: A guide for students and practitioners (pp. 209-223). Chichester: Wiley.

Lehmann, S., Heiervang, E. R., Havik, T., \& Havik, O. E. (2014). Screening foster children for mental disorders: properties of the strengths and difficulties questionnaire. PloS one, 9(7), e102134.

Luyten P, Mayes LC, Nijssens L, Fonagy P. (2017) The parental reflective functioning questionnaire: Development and preliminary validation. PLOS ONE, 12(5):e0176218.

Milburn, N. L., Lynch, M., \& Jackson, J. (2008). Early identification of mental health needs for children in care: a therapeutic assessment programme for statutory clients of child protection. Clinical child psychology and psychiatry, 13(1), 31-47.

NICE (2010) Looked-after children and young people. Public Health Guideline [PH28] Manchester: National Institute of Health and Care Excellence. https://www.nice.org.uk/guidance/ph28 


\section{SDQ LAC MENTAL HEALTH}

Ratnayake, A., Bowlay-Williams, J., \& Vostanis, P. (2014). When are attachment

difficulties an indication for specialist mental health input? Adoption \& Fostering, 38(2), 159-170.

SCIE (2017) Improving mental health support for our children and young people. Expert Working Group final report. https:/www.scie.org.uk/children/care/mental-health/report

Streiner, D. L., Cairney, J. (2007). What's under the ROC? An introduction to Receiver Operating Characteristics Curves. The Canadian Journal of Psychiatry, 52, 121- 128.

Tarren-Sweeney, M. (2018). Mental Health Screening and Monitoring for Children in Care: A Short Guide for Children's Agencies and Post-adoption Services. Routledge.

Wassall, S.L.V., Golding, K. S., \& Barnbrook, E. (2011). Reliability of the Carers Questionnaire for foster carers and adoptive parents. Unpublished document.

Van der Kolk, B.A. (2005) Developmental Trauma Disorder. Psychiatric Annals, 35, 5, p. 401-408.

Youth in Mind (2016) Instructions in English for scoring by hand SDQs for 4-17 year olds. https://sdqinfo.org/py/sdqinfo/c0.py 
SDQ LAC MENTAL HEALTH

\section{Acknowledgements}

This paper outlines a study which was undertaken as part of an independent research funded by the National Institute for Health Research (NIHR) Research for Patient Benefit (RfPB) themed grant (grant number: PB-PG-0614-34079) and supported by the NIHR Clinical Research Network (CRN). The views expressed are those of the authors, and not necessarily those of the NHS, the NIHR or the Department of Health.

The authors would like to thank the CAMHS targeted team therapists who were interviewed for the study, and CAIS Islington for consultation. 